\title{
Navigating Radiation Therapy During COVID-19 Using YouTube as a Source of Information
}

\author{
Zhang Hao Jim $\mathrm{Li}^{1} \cdot$ Inhwa $\mathrm{Kim}^{2} \cdot$ Meredith Giuliani ${ }^{3,4} \cdot$ Paris-Ann Ingledew ${ }^{5,6}$
}

Accepted: 2 January 2022 / Published online: 14 January 2022

(c) The Author(s) under exclusive licence to American Association for Cancer Education 2022

\begin{abstract}
The COVID-19 pandemic brought considerable change to the practice of radiotherapy. In the meantime, patients are increasingly turning to online resources for health information, with YouTube being one of the biggest platforms. However, little is known about what information is being disseminated to cancer patients about radiotherapy in the context of COVID19. Therefore, this study aims to characterize and assess YouTube videos on radiotherapy during COVID-19. A YouTube search using the terms "Radiation therapy COVID-19", "Radiation therapy coronavirus", "Radiotherapy COVID-19", and "Radiotherapy coronavirus" was completed using a clear-cache web browser. The top 50 videos were collected from each search. After applying pre-determined exclusion criteria, each video was assessed for general parameters, source, and content. Two raters were used to ensure interrater reliability. One hundred five unique videos resulted from the four searches. Ninety-eight per cent were published in the last year. The median video length was 6 min and $54 \mathrm{~s}$, and the median number of views was 570. Most videos were from the USA (58\%). The majority of videos were published by a commercial channel (31\%), non-profit organization (28\%), or healthcare facility (26\%). Forty-two per cent of the videos covered a topic related to radiotherapy during the pandemic. Bias was identified in $6 \%$ of videos. YouTube information on radiotherapy during COVID-19 is non-specific and can be misleading. The results of this study highlight the need for healthcare providers to proactively address patient information needs and guide them to appropriate sources of information.
\end{abstract}

Keywords Patient education · Online health information · Quality assessment $\cdot$ Radiotherapy $\cdot$ Radiation therapy $\cdot$ COVID19

Paris-Ann Ingledew

pingledew@bccancer.bc.ca

1 Faculty of Medicine, University of British Columbia, 317 2194 Health Sciences Mall, Vancouver V6T 1Z3, Canada

2 Faculty of Medicine, Dalhousie University, 5849 University Ave, Halifax B3H 4R2, Canada

3 Department of Radiation Oncology, University of Toronto, 149 College St \#504, Toronto M5T 1P5, Canada

4 Princess Margaret Cancer Centre, 610 University Ave, Toronto M5G 2C1, Canada

5 Division of Radiation Oncology, Department of Surgery, University of British Columbia, 2775 Laurel Street, Vancouver V5Z 1M9, Canada

6 BC Cancer Agency Vancouver Centre, 600 West 10th Avenue, Vancouver V5Z 4E6, Canada

\section{Introduction}

Radiotherapy (RT) is one of the main modalities of cancer treatment. The COVID-19 pandemic has transformed the way many medical services are offered, and RT is no exception. To reduce the risk of COVID-19 transmission, some clinicians have been deviating from standard practice by offering patients less frequent, but higher doses of radiation, a process called hypofractionation [1,2]. Although hypofractionation is not appropriate for all cancer treatments, certain guidelines have endorsed this practice in limited situations [3]. Concurrently, patient load, total treatment courses, and clinical trial enrolment have also decreased in the wake of the pandemic, possibly due to anxiety surrounding exposure to COVID-19 in the healthcare setting [1, 2, 4]. This raises concerns as there are deleterious consequences of delaying or forgoing RT for cancer, such as increased risk of progression and recurrence [5]. Therefore, the consensus statement by the European Society for Radiotherapy and 
Oncology (ESTRO) and the American Society for Radiation Oncology (ASTRO) recommended RT for all eligible patients who do not have COVID-19, provided that resources are available [3].

Another major change to the radiotherapy process is the transitioning of routine clinical services to a telemedicine format, which has fundamentally changed the nature of patient-physician interactions. Although telemedicine has been shown to improve certain psychosocial outcomes associated with cancer [6], it is also known that cancer patient and their families often have unmet information needs and the telemedicine process may come across as impersonal when delivering health-related information $[6,7]$. Thus, it is not surprising that online health-information seeking behaviour has increased at the beginning of the pandemic, including among cancer patients $[8,9]$.

In the meantime, YouTube is the world's second most visited website behind Google [10]. With over two billion regular users and one billion hours of videos watched daily, it has the potential to provide easy access to medical information free-of-charge [11]. As the COVID-19 pandemic rose to prominence in early 2020 , a large body of unregulated online information regarding the pandemic appeared; the majority of these information sources was of poor quality, difficult for the average reader to understand, and encumbered with misinformation [12]. Unfortunately, this problem was not unique to traditional text-based websites. Information on YouTube videos was also shown to be of generally low quality, and prone to misinformation [13]. Despite these concerns surrounding the quality of YouTube information on COVID-19 and recent changes in radiotherapy delivery, no literature exists on what information patients are receiving on YouTube about radiotherapy during the pandemic. Since YouTube has immense potential to aid or hinder cancer treatment and public health efforts, clinicians should be aware of what information is available to patients in order to better guide counselling discussions. Therefore, we endeavour to systematically assess the characteristics of YouTube videos on radiotherapy during the pandemic, with a particular focus on video origin, content, and its relevance to COVID-19.

\section{Materials and Methods}

\section{Search Protocol}

A clear-cache Google Chrome browser in incognito mode was used to search all videos to mitigate unintentional bias from previous search histories or geographical location. Four separate YouTube searches were completed on 24 January 2021 using the following terms, respectively: "radiation therapy COVID-19", "radiation therapy coronavirus", "radiotherapy COVID-19", and "radiotherapy coronavirus". The search results were sorted by "Relevance", which is the default setting on YouTube.

\section{Data Collection}

The first 50 videos were recorded from each of the four searches. After removing duplicates, the following pre-determined exclusion criteria were then applied to the remaining videos: language other than English, length longer than one hour, and publication date before January 2020 [Fig. 1]. The cut-off of January 2020 was chosen as this was when the infectious agent was determined to be a novel coronavirus; hence, our search terms would only be valid after this time [14].

\section{Video Parameters}

As there is not yet a widely accepted tool designed for analyzing videos in the existing literature, a systematic coding
Fig. 1 Flow diagram of YouTube search results and application of exclusion criteria

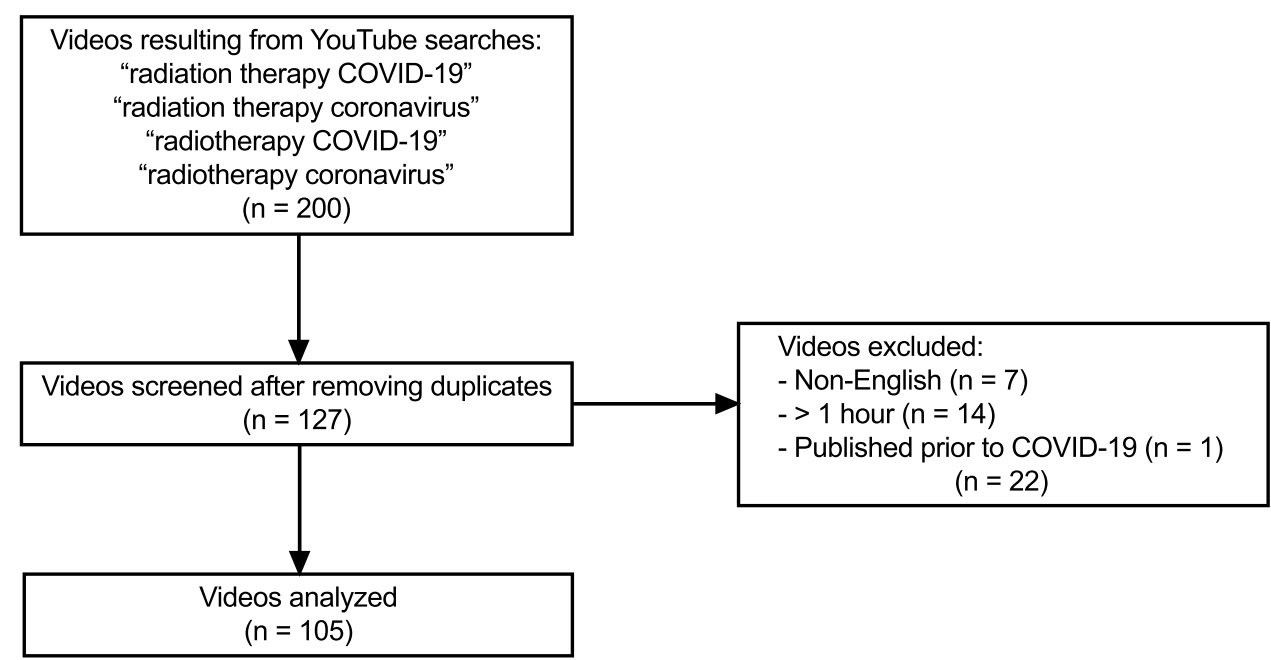


tool was developed by our research team and applied to the current set of videos in this study $[15,16]$. Each video was assessed for three general parameters revolving around the video itself, the publisher, and the content of the video. Information collected about the video included number of views, date of publication, number of likes and dislikes, Video Power Index (VPI, the product of number of views and the like/dislike ratio), and video length. Information regarding the publisher included the channel name, country of origin, number of subscribers, publisher affiliation (healthcare facility, non-profit, commercial, educational institution, government, personal), and presenter identification (patient, physician, allied health professional, not identified, other). Information about the content included presence of subtitles, media type, target audience, topic, number of comments, comment moderation, advertisements, and identifiable bias. Media type refers to the main style of video used to convey the information-namely physician interview, patient interview, live video of the treatment process, computer graphics, or lecture presentations. The target audience is subjectively determined based on the level of technical language used, comment moderation is operationalized as the host channel responding to at least one comment, and identifiable bias is operationalized as the promotion of unproven treatments for either cancer or COVID-19. Specifically, if a video's topic is related to radiotherapy in the context of the COVID-19 pandemic, it would be considered "relevant", and if otherwise, "less relevant". In general, these parameters reflect previously validated tools for text-based health information, such as the HON Code and the JAMA benchmarks $[17,18]$. One notable addition was the VPI, which is a commonly used objective measure for video popularity with no implications on informational quality [19]. To elaborate on the VPI, a video with many views, likes, and few dislikes will have a high VPI. It has no theoretical maximum. All these parameters could be easily found on YouTube itself. In the case that the publisher affiliation or target audience is not immediately clear, two reviewers would determine the most appropriate category through discussion.

\section{Statistical Analysis}

The validity of coding was ensured through two independent assessments using the video coding tool on a random sample of 10 videos by two reviewers. Any discrepancies were resolved by discussion. Inter-rater reliability was deemed acceptable as the kappa score for the initial sample of 10 videos was $>0.7$; the remaining videos were then assessed by one reviewer independently. Had the kappa score been $<0.7$, indicating poor inter-rater agreement, the reviewers would have clarified points of discrepancy and re-evaluated the inter-rater reliability with another random sample of $10 \mathrm{web}-$ sites until the resulting kappa score is $>0.7$. The data were analyzed with both descriptive statistics (i.e. measures of central tendency) and Fisher's exact test to determine if videos from commercial sources were more likely to be biased.

\section{Results}

\section{Video Characteristics}

Two hundred videos were recorded from the four searches. After removing the duplicates and applying the exclusion criteria, there were 105 unique English-language videos remaining. They were all published between 23 January 2020 and 11 January 2021. Ninety-eight per cent of the videos were published within the last year; the distribution of video currency (how recently published) is shown in Table 1 . The number of views followed a positively skewed distribution, with a skewedness of 6.29. The range was from 6 to $11,690,614$ views, and the median was 566. Of note, the three most watched videos had more views than the other videos combined. Similarly, the video power index (VPI) also followed a positively skewed distribution, with a skewedness of 5.67. The median VPI was 4.29. The median video length was $7 \mathrm{~min}$ and $6 \mathrm{~s}$.

\section{Video Source}

The majority of videos were from the USA (58\%) and the UK (17\%). A small portion of videos were from India (8\%), Australia (3\%), Italy (2\%), Germany (2\%), and Switzerland (2\%). The remaining $8 \%$ of videos were from New Zealand, Canada, Ireland, Belgium, Sweden, Kenya, Indonesia, Turkey, and of unknown origin [Table 1].

The majority of videos were published by a commercial channel (31\%), non-profit organization (28\%), or healthcare facility (26\%). The remaining videos were published by a personal channel (10\%), educational institution (4\%), or government-affiliated channel (2\%) [Table 1].

The main presenter in these videos were physicians (71\%). Other presenters include patients (6\%) and allied health professionals such as nurses, radiotherapists, and pharmacists (5\%). Eight per cent of the videos did not have a clearly identified presenter (i.e. slideshows or voiceovers). Ten per cent of the videos were presented by a group of people categorized as "Other", which included PhD scientists, news anchors, engineers, and chief executive officers [Table 1].

\section{Video Content}

The majority of the videos were interviews with physicians (59\%) or lecture-style presentations (20\%). The remaining videos were either patient interviews (4\%), live videos (8\%), 
Table 1 Video parameters

\begin{tabular}{|c|c|}
\hline Parameter & $\mathrm{N}(\%)$ \\
\hline \multicolumn{2}{|l|}{ Number of views } \\
\hline$<10$ & $1(1 \%)$ \\
\hline $10-100$ & $14(13 \%)$ \\
\hline $100-1,000$ & $43(41 \%)$ \\
\hline $1,000-10,000$ & $20(19 \%)$ \\
\hline $10,000-00,000$ & $10(10 \%)$ \\
\hline $100,000-1,000,000$ & $8(8 \%)$ \\
\hline $1,000,000+$ & $9(9 \%)$ \\
\hline \multicolumn{2}{|l|}{ Currency } \\
\hline$<3$ months & $3(3 \%)$ \\
\hline $3-6$ months & $14(13 \%)$ \\
\hline 6-9 months & $35(33 \%)$ \\
\hline 9-12 months & $51(49 \%)$ \\
\hline$>12$ months & $2(2 \%)$ \\
\hline \multicolumn{2}{|l|}{ Source of video } \\
\hline USA & $61(58 \%)$ \\
\hline UK & $18(17 \%)$ \\
\hline India & $8(8 \%)$ \\
\hline Australia & $3(3 \%)$ \\
\hline Italy & $2(2 \%)$ \\
\hline Germany & $2(2 \%)$ \\
\hline Switzerland & $2(2 \%)$ \\
\hline New Zealand & $1(1 \%)$ \\
\hline Canada & $1(1 \%)$ \\
\hline Ireland & $1(1 \%)$ \\
\hline Belgium & $1(1 \%)$ \\
\hline Sweden & $1(1 \%)$ \\
\hline Kenya & $1(1 \%)$ \\
\hline Indonesia & $1(1 \%)$ \\
\hline Turkey & $1(1 \%)$ \\
\hline Unknown & $1(1 \%)$ \\
\hline \multicolumn{2}{|l|}{ Publisher affiliation } \\
\hline Commercial & $33(31 \%)$ \\
\hline Non-commercial & $72(69 \%)$ \\
\hline Healthcare facility & $27(26 \%)$ \\
\hline Non-profit & $29(28 \%)$ \\
\hline Educational institution & $4(4 \%)$ \\
\hline Government & $2(2 \%)$ \\
\hline Personal & $10(10 \%)$ \\
\hline \multicolumn{2}{|l|}{ Presenter } \\
\hline Patient & $6(6 \%)$ \\
\hline Physician & $75(71 \%)$ \\
\hline Allied health professional & $5(5 \%)$ \\
\hline Other & $11(10 \%)$ \\
\hline Not identified & $8(8 \%)$ \\
\hline
\end{tabular}

or computer graphics (9\%) [Fig. 2]. 89\% of videos featured subtitles. Although the majority of videos were targeted to patients (68\%), a significant portion of videos were directed towards healthcare professionals (32\%). For example, a conference presentation would be considered a "lecture-style presentation" targeted towards healthcare professionals, whereas a virtual tour through a cancer treatment centre would be a "live video" targeted towards a patient audience.

The major relevant themes that were featured in the videos include safety of radiotherapy (RT) during the pandemic (main topic in $13 \%$ of videos), changes to RT during the pandemic (11\%), and information for specific cancers requiring radiation during the pandemic $(17 \%)$. Specifically, frequent topics from the theme of safety included safety measures for in-person visits and addressing patient concerns regarding telemedicine. Meanwhile, hypofractionation and delays to treatment were common topics under the theme of changes to RT during the pandemic. All 44 videos which discussed one of the above relevant themes recommended patients to start/continue RT as medically indicated; however, 14 videos also acknowledged that treatment may be delayed for certain scenarios (e.g. when there is no evidence that a delay will impact outcomes). Less relevant themes from our search results included general COVID-19 information (33\%), general RT information (5\%), and non-RT related cancer information in the context of the pandemic (11\%). The remaining $9 \%$ of videos were categorized as "Other." It should be noted that all of the ten most watched videos featured a "less relevant" theme. In total, $42 \%$ of videos featured a relevant theme whereas $58 \%$ did not.

Six videos $(6 \%)$ were identified as containing identifiable bias of some sort, of which five were from commercial channels and one was from a personal channel. Commercial videos were statistically more likely to be biased compared to non-commercial videos (Fisher's exact test $P=0.01$ ). It should be noted that none of these six videos contained relevant information for radiation therapy during the pandemic. Examples of biased information include using elderberries to treat COVID-19 infection, and promoting the consumption of traditional spices to boost one's immune system. Eighteen per cent of the videos contained at least one advertisement from YouTube. A small portion (16\%) of the videos had comment moderation, as evidenced by the host channel responding to at least one comment. Thirty-one per cent of the videos had no comment moderation at all, $17 \%$ of the videos had disabled comments, and the remaining $35 \%$ of the videos had no comments, so their moderation was considered unknown.

\section{Discussion}

The COVID-19 pandemic has caused unforeseen disruptions to radiotherapy (RT) delivery across the world. Previous patterns of practice have evolved to incorporate telemedicine, and in some situations, altered RT schedules 
Fig. 2 Distribution of included videos by media type

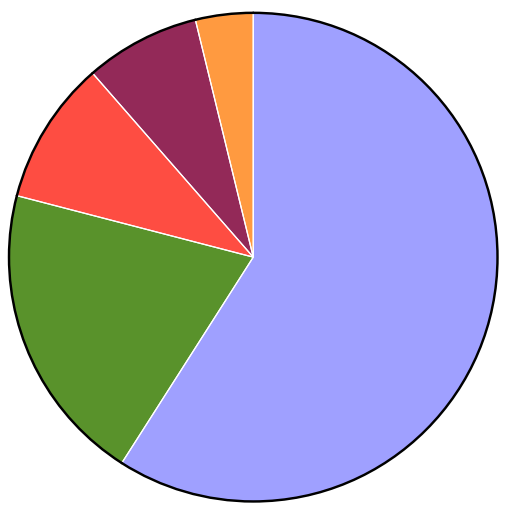

59\% Physician interview $20 \%$ Lecture-style presentation $8 \%$ Live video 9\% Computer graphics 4\% Patient interview

\section{Total=105}

such as hypofractionation were implemented to minimize COVID-19 exposure risk [1-3]. At the same time, patients are increasingly using the Internet for health-related information $[8,9]$. However, despite these changes to RT during the pandemic, it was not clear what information patients have access to online. Therefore, we felt compelled to systematically examine the quality of YouTube videos regarding RT in the context of COVID-19.

As with any health information media, having up-to-date information is key to helping patients make informed decisions. Out of the 105 videos selected for analysis, the vast majority were published in the 12 months leading up to January 2021, which was expected as the COVID-19 pandemic only came into the public eye in late 2020 and early 2021. Although the currency for this sample of videos would have been considered adequate using conventional standards for evaluating online health information, which is for the information to be published within the past two years [20], in the context of the rapidly evolving COVID-19 pandemic, one could argue that this is too long of a timeframe for information to stay up-to-date. Although no standards exist for how information currency should be evaluated during an evolving pandemic, it would not be unreasonable to suggest a narrower timeframe of nine months for information to be considered up-to-date, as the WHO guidelines for pandemic restrictions and adjustments to health services to reduce the risk of COVID-19 exposure were released approximately nine months prior to the search, around March-April 2020 [21]. If this metric was used, then $50 \%$ of videos would be up-to-date at the time the search was performed. While there is space for improvement, this rate of currency would still be considered relatively high compared to other cancer-related topics [22].

The majority (71\%) of the videos were presented by physicians. While this is encouraging in that physician-delivered videos have been shown to be of higher quality than nonphysician videos [23], it should also be noted that only $6 \%$ of videos featured patient presenters. This implies that it is difficult for patients and their families to hear about lived experiences of others and to receive peer encouragement, which have been shown to be therapeutic coping mechanisms, even in the online setting [24]. Although previous psychological research has not shown that personal stories help patients make more informed decisions, there is evidence that it could assist with improving information recall and future motivation to discuss decision-making [25]. Therefore, as social distancing restrictions gradually ease, it is important that patients are offered the chance to recount their experiences for others who may be following their footsteps.

Commercialization of health information is a known predictor of bias and poorer information quality, as authors face a conflict of interest between presenting accurate information and marketing commercial services [26]. This was reaffirmed in our study; although only six videos from the search had identifiable bias, they were more likely to be from commercial sources than non-commercial sources. Interestingly, none of the videos from an educational institution, healthcare facility, non-profit, or government source contained grossly biased information. The only biased noncommercial video was from a personal source. Therefore, healthcare professionals should be aware of potential biases present in commercial sources and take this into account when prescribing online health information.

In terms of video content, it is surprising that only $42 \%$ of videos featured information relevant to $\mathrm{RT}$ in the context of the COVID-19 pandemic. A possible explanation for this is that our search combined the terms of "radiation therapy" or "radiotherapy" with "COVID-19" or "coronavirus". Thus, many videos applicable to only one of the terms have been captured by the search and diluted the overall results. Another, perhaps more likely explanation, is that there is simply a deficit of informational videos related to our relatively niche research inquiry. Regardless of the reason, the key point is that patients conducting YouTube searches 
independently may not always find relevant information on this topic. It should therefore be the onus of healthcare providers to directly educate patients or guide them to reliable online resources regarding RT during COVID-19.

Effective comment moderation is essential to ensuring that patients and families can ask questions and share their experiences in a safe environment. Only $16 \%$ of videos had visible comment moderation, as evidenced by the host channel replying to at least one comment. The remaining videos either had no comment moderation (31\%), disabled comments (17\%), or no comments (35\%). The lack of comment moderation potentially exposes patients to misinformation, which could cause harm by creating treatment hesitancy or influencing patients to pursue inappropriate therapy. Although disabling comments may seemingly protect viewers from this, it may come across as negligent and closes an avenue for patients to share their lived experiences, which is another critical drawback as cancer patients and their families frequently report psychosocial distress [27]. Finally, while we cannot be certain whether the 35\% of videos with no comments would be moderated, the near 1:2 ratio between moderated and unmoderated videos suggests that the majority of those with no comments would likely be unmoderated as well. Given the overall lack of well-moderated comment sections, it is our recommendation that healthcare providers refer patients to structured support groups and advise them to be wary of misinformation that they may see online.

One limitation to our study is that it only represents a snapshot in time. As new videos are uploaded constantly unto YouTube, which also may change its algorithm for displaying videos over time, our search results are likely to be different compared to the same search performed a few months earlier or later. Furthermore, our study focused more on objective characteristics instead of a subjective quality assessment. While this may have reduced the risk of unintentional reviewer bias, it also limits the conclusions that we were able to draw regarding video quality. Future studies could examine the quality of YouTube videos on this topic, especially focusing on informational accuracy.

The results of our study show that most YouTube videos regarding RT during the COVID-19 pandemic did not contain identifiable bias; those that did were more likely to be from commercial channels. However, video currency was not ideal, most videos captured from our search were factually unrelated to pandemic-era RT, comment moderation was often absent, and a significant portion of relevant videos endorsed delaying RT. Thus, it is imperative that healthcare providers proactively ensure that patient information needs about this topic are met via direct education or an online information prescription. Additionally, patient concerns regarding treatment safety should be addressed to prevent unnecessary delays which may result in worsening disease progression. These are critical steps in removing information barriers facing patients.

Author Contribution All authors contributed to the study conception and design. Data collection and analysis were performed by Inhwa Kim and Jim Li. The first draft of the manuscript was written by Jim Li and Inhwa Kim, and all authors contributed to editing the manuscript. All authors have read and approved the final manuscript.

Data Availability Available upon reasonable request.

Code Availability Not applicable.

\section{Declarations}

Ethics Approval Not applicable, as no human or animal subjects were involved.

Consent to Participate Not applicable.

Consent for Publication Not applicable.

Competing Interests The authors declare no competing interests.

\section{References}

1. Roberge D, Delouya G, Bohigas A, Michalowski S (2021) Catching the wave: quantifying the impact of COVID on radiotherapy delivery. Curr Oncol 28:152-158

2. Spencer K, Jones CM, Girdler R et al (2021) The impact of the COVID-19 pandemic on radiotherapy services in England, UK: a population-based study. Lancet Oncol 22:309-320

3. Guckenberger M, Belka C, Bezjak A et al (2020) Practice recommendations for lung cancer radiotherapy during the COVID-19 pandemic: an ESTRO-ASTRO consensus statement. Int J Radiat Oncol Biol Phys 107:631-640

4. De B, Kaiser KW, Ludmir EB et al (2021) Radiotherapy clinical trial enrollment during the COVID-19 pandemic. Acta Oncol (Madr) 60:312-315

5. Nagar H, Formenti SC (2020) Cancer and COVID-19-potentially deleterious effects of delaying radiotherapy. Nat Rev Clin Oncol 17:332-334

6. Cox A, Lucas G, Marcu A et al (2017) Cancer survivors' experience with telehealth: a systematic review and thematic synthesis. J Med internet Res 19:e11

7. Wang T, Molassiotis A, Chung BPM, Tan J-Y (2018) Unmet care needs of advanced cancer patients and their informal caregivers: a systematic review. BMC Palliat Care 17:1-29

8. Vanderpool RC, Huang GC, Mollica M et al (2021) Cancer information-seeking in an age of COVID-19: findings from the National Cancer Institute's Cancer Information Service. Health Commun 36:89-97

9. Giuliani M, Papadakos T, Papadakos J (2020) Propelling a new Era of patient education into practice - cancer care post-COVID19. Int J Radiat Oncol Biol Phys 108:404-406

10. Alexa The top 500 sites on the web. https://www.alexa.com/topsi tes. Accessed 28 Jun 2021

11. YouTube (2021) YouTube for Press. https://www.youtube.com/ intl/en-GB/about/press/. Accessed 7 Jul 2021 
12. Zhao X, Fan J, Basnyat I, Hu B (2020) Online Health Information Seeking Using “\# COVID-19 Patient Seeking Help" on Weibo in Wuhan, China: Descriptive Study. J Med Internet Res 22:e22910

13. Arya R, Ichikawa T, Callender B et al (2020) Communicating the external beam radiation experience (CEBRE): perceived benefits of a graphic narrative patient education tool. Pract Radiat Oncol 10:e219-e226

14. World Health Organization (2020) WHO Statement regarding cluster of pneumonia cases in Wuhan, China. https://www.who. int/china/news/detail/09-01-2020-who-statement-regarding-clust er-of-pneumonia-cases-in-wuhan-china. Accessed 24 Jun 2021

15. Gabarron E, Fernandez-Luque L, Armayones M, Lau AYS (2013) Identifying measures used for assessing quality of YouTube videos with patient health information: a review of current literature. Interact J Med Res 2:e2465

16. Drozd B, Couvillon E, Suarez A (2018) Medical YouTube videos and methods of evaluation: literature review. JMIR Med Educ 4:e8527

17. Health on the Net Foundation (2019) Discover the 8 principles of the HONcode in 35 languages. https://www.hon.ch/cgi-bin/ HONcode/principles.pl?English. Accessed 13 Jan 2021

18. Silberg WM, Lundberg GD, Musacchio RA (1997) Assessing, controlling, and assuring the quality of medical information on the Internet: Caveant lector et viewor-Let the reader and viewer beware. J Am Med Assoc 277:1244-1245. https://doi.org/10. 1001/jama.1997.03540390074039

19. Erdem MN, Karaca S (2018) Evaluating the accuracy and quality of the information in kyphosis videos shared on YouTube. Spine (Phila Pa 1976) 43:E1334-E1339
20. Li G (2007) The median age technique for assessing currency of consumer health information monographic collections in public libraries. J Med Libr Assoc 95:89

21. World Health Organization (2021) Timeline: WHO's COVID-19 Response. https://www.who.int/emergencies/diseases/novel-coron avirus-2019/interactive-timeline\#! Accessed 9 Jul 2021

22. Sahin AN, Sahin AS, Schwenter F, Sebajang H (2019) YouTube videos as a source of information on colorectal cancer: what do our patients learn? J Cancer Educ 34:1160-1166

23. Kuru T, Erken HY (2020) Evaluation of the quality and reliability of YouTube videos on rotator cuff tears. Cureus 12:

24. Klemm P, Bunnell D, Cullen M et al (2003) Online cancer support groups: a review of the research literature. CIN Comput Informatics, Nurs 21:136-142

25. Bekker HL, Winterbottom AE, Butow P et al (2013) Do personal stories make patient decision aids more effective? A critical review of theory and evidence. BMC Med Inform Decis Mak 13:1-9

26. Asafu-Adjei D, Mikkilineni N, Sebesta E, Hyams E (2019) Misinformation on the internet regarding ablative therapies for prostate cancer. Urology 133:182-186

27. Carlson LE, Zelinski EL, Toivonen KI et al (2019) Prevalence of psychosocial distress in cancer patients across 55 North American cancer centers. J Psychosoc Oncol 37:5-21

Publisher's Note Springer Nature remains neutral with regard to jurisdictional claims in published maps and institutional affiliations. 